\title{
Case Report: Postmortem Incidental Ependymal Cyst Opened to the Extracranial Area
}

\author{
Gizem Muratoglu*, Ibrahim Ortanca, Muhammed Mustafa Arslan \\ Department of Forensic Medicine, Mustafa Kemal University Medical Faculty, Hatay/Turkey \\ *Corresponding Author: Gizem Muratoglu, Department of Forensic Medicine, Mustafa Kemal \\ University Medical Faculty, Hatay/Turkey, Email: giz.muratoglu@gmail.com
}

\begin{abstract}
Glioependymal cysts are rare and generally remain asymptomatic. But they may give symptoms according to the localization and size of the cyst.

We present a case who died due to aortic rupture with an ependymal cyst in parietal lobe opened to the extracranial area that was incidentally found in the autopsy, which has not been published in the literature before.

Eighty-three-year-old malewas found dead in his bathroom. In the autopsy, 1200ml blood was found in the pericardial cavity with anaortic laceration. Death was due to cardiac tamponade resulted from an aortic rupture. In addition to this, a defect on the parietal bone was found. Two connected cystic formations were seen on the right parietal lobe of the brain, just beneath the bone defect. In the histopathological examination, the cystic lesion was compatible with the glioependymal cyst.

Ependymal cyst remained asymptomatic despite the destruction of the skull. And till his death, no other cranial imaging was performed that could have detect the cyst before. Unlike the anamnesis taken from his relatives, the findings supporting the existence of chronic diseases made us think that he was not having regular health controls probably due to his low socioeconomic status.
\end{abstract}

Keywords: ependymal cyst, cranial defect, autopsy, incidental

\section{INTRODUCTION}

Symptomatic intracranial cysts are the lesions rarely seen in the clinic. The increasing use of imaging methods has facilitated the diagnosis of intracranial cysts [1]. Among intracranial cysts, the ratio of glioependymal (ependymal) cysts is less than $1 \%$. The ependymal cysts that mainly settle as intraparenchymal are most frequently seen around ventricle in the frontal and parietal area. They are congenital lesions consisting of ependymal cells islands that settle into the developing white matter by separating from the neural tube during embryonic development. They are properly limited, unilocular, include transparent liquid like CSF. They are surrounded by ependymal (columnar epithelium) or choroid plexus cells (low cubic epithelium). Most of the patients remain asymptomatic but may also show symptom according to the localization and size of the cyst $[2,3]$.

Here we present a case of postmortem incidental ependymal cyst opened to the extracranial area which has not been published in the literature before.

\section{CASE}

Eighty-three-year-old male patient lives with his wife and daughter. He was found dead next to the water closet in the bathroom of the house in the morning. In the anamnesis taken from his relatives, it was stated that he had no health problem out of scoliosis, he went to work every morning, he came home towards evening, and his socio-economic level was low. In the examination of his medical records, it was seen that the medical examinations of the patient was performed with the pre-diagnosis of renal failure but he did not have neither further evaluation nor diagnosis of a chronic disease.

During dead examination, $3 \times 2 \mathrm{~cm}$ purple ecchymosis under the right eye, $5 \mathrm{~cm}$ scar of old wound on the right leg and $3 \times 2 \mathrm{~cm}$ scar of old wound on the outer side on the left leg were observed, the soft mass in the right temporoparietal area was palpated. 
During autopsy, a serous fluid flowed from the right parietal region while removing the scalp and periosteum was detected. The bone defect which was $5 \mathrm{~cm}$ in length and the widest part of which was $2 \mathrm{~cm}$ was observed in the right parietal bone (Figure 1). Brain, cerebellum, brain stem were weighed $1400 \mathrm{~g}$. No epidural, subdural and subarachnoid hemorrhage was detected. It was determined that the dura mater and the inner lamina in the area of $6 \mathrm{~cm}$ around the defect on the right parietal bone were destructive. In this destructive region, papillary formations with a structure different from the bone tissue were observed (Figure 2). Two cystic formations of $3 \mathrm{~cm}$ in diameter which were connected with each other were determined on the right parietal lobe of the brain, and it was found that the arachnoid membrane lost its integrity; the cysts were associated with the subdural space (Figure 3). In basilar arteries, 50-60\% occlusive atherosclerotic changes were locally seen. In the brain cross-sections, no pathological feature was observed out of edema and hyperemia. It was determined that

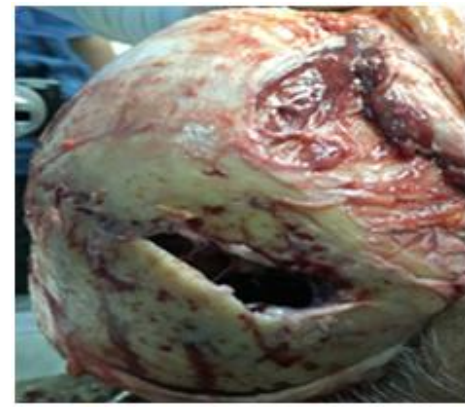

Figure1. The outside view of the right parietal bone

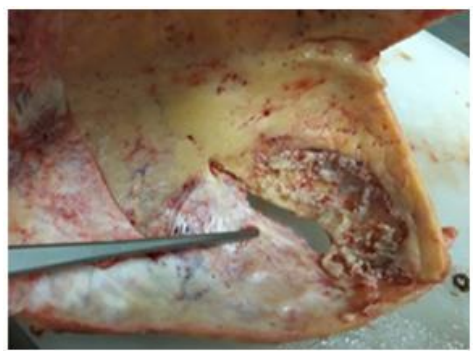

Figure2. The inside view of the right parietal bone

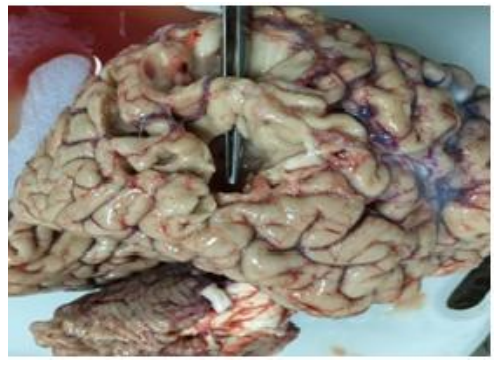

Figure3. Two connected cystic formations on the right parietal lobe there were $800 \mathrm{cc}$ sero-hemorrhagic liquid in the right chest cavity and free blood around $1200 \mathrm{cc}$ in the pericardial cavity. The lungs were anthracotic. The hearth was removed with thoracic aorta, and they were weighed as 1100 gr. Up to $80 \%$ occlusive atherosclerotic changes were observed in the coronary arteries. The tricuspid valve was measured as $14.5 \mathrm{~cm}$, pulmonary valve as $9 \mathrm{~cm}$, mitral valve as $12.5 \mathrm{~cm}$, aortic valve as $7.5 \mathrm{~cm}$, left ventricular wall thickness as $2.2 \mathrm{~cm}$, septum as $2 \mathrm{~cm}$. An aneurism on the ascending aorta and $3 \mathrm{~cm}$ fullthickness laceration just above the aortic valve were observed (Figure4). There were common hard calcified plaques in the aorta. There were nodular changes in the liver surface and cross sections. The right kidney surface was irregular, its sections were congested, the cortex-medulla limit could not be distinguished, the left kidney was small by volume, perinephric fat tissue was increased, cysts were seen in the sections. It was concluded that the death of the person occurred as a result of cardiac tamponade due to aortic rupture.

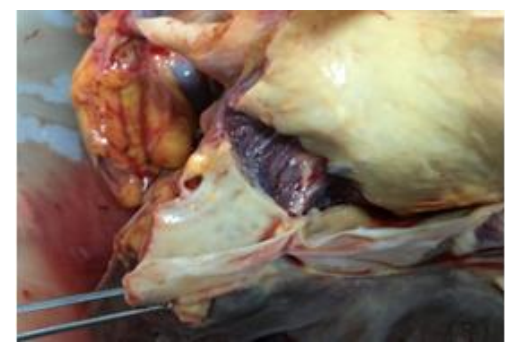

Figure4. Aortic rupture above the aortic valve

In the histopathological examination of the cystic formation in the brain tissue, it was determined that there was gliosis tissue around the simple cystic lesion. Cyst epithelium GFAP was stained positive with $\mathrm{S} 100$ and negative with EMA. In the immunohistochemical study performed in the area gliosis, it was observed that the glial cells in the normal histology were positive with GFAP, S100, and CD57.Ki67 proliferation index was evaluated as $0 \%$. It was thought that the cystic lesion could mostly be compatible with the glioependymal cyst of the central nervous system cysts (Figure 5).



Figure5. The cyst limited by cubic epithel and surrounding with gliozis (H/Ex200) 


\section{DISCUSSION AND CONCLUSION}

The ependymal cysts usually progress a symptomatically, however may cause symptoms according to their locations. Common clinical presentation occurs with seizure, syncope and headache. It was reported that neurological deficits such as hemiparesis, hemihypoesthesia, hemianopia, ataxia, memory or personality changes, confusion, drowsiness, headache, mental disorders could be seen as more rare findings [4-7].

In the older literature when the imaging techniques were not widely used, we see that many cases were admitted to health institutions with various complaints got different diagnosis, but later ependymal cyst were detected in the autopsy $[7,8]$. In current publications, the brain cyst cases that can be diagnosed with imaging methods and pathology have been presented, differential diagnosis of the cysts and treatment methods have been discussed [3,9,-12].It was stated in a study that the frontal region cysts were presented with headache and weakness in 3 female patients aged 52-60-64 [7], and it was also stated in another study that the ependymal cyst in the left parietal lobe caused seizure and left 6th nerve paresis in a 74-year-old female patient [8]. It was reported that the ependymal cyst in the parietotemporal region presented with macrocephaly in a three-month baby [5], the cyst settled in front parietal caused left weakness and headache in a 20-year-old male patient [2], and a 42-year-old male patient with ependymal cyst in the occipital region had headaches and seizures [4].

It is known that a space-occupying formation in the brain, independently of the localization, may cause headache and epileptic seizures along with the increased intracranial pressure. The focal neurologic deficits can also be seen beside these common symptoms. The contra lateral cortical sensory abnormalities, neglect syndrome, Gerstmann's syndrome, perceptual aphasia, difficulty in complex motor functions, contra lateral inferior quadrantanopia or hemianopia can be seen in the parietal lobe lesions [13, 14].

In our patient, the parietal lobe-settled cyst of $3 \mathrm{~cm}$ in diameter remained asymptomatic, and diagnosis was not made with any imaging method. The asymmetry due to the cyst and bone defect were externally noticeable, however according to the anamnesis taken from his relatives, it was interesting for us that the patient did not seek for any medical help. When it was considered that the ependymal cysts were congenital, it was thought that imaging of the head region of the patient was not performed until his death, he was not followed in terms of health, and the low socioeconomic status of patient affected this situation. In addition, the absence of complaints such as headache, epileptic seizures and sensory abnormalities also made it difficult to make diagnosis. Another point is, besides the brain cyst, other findings such as effusion in the right chest cavity, cardiac hypertrophy, coronary atherosclerosis, the pathological changes in renal morphology supporting the existence of chronic diseases were found in this case.

This glioependymal cyst opening from parietal lobe of the brain to the extracranial area with a destructive lesion in the dura mater and bone that was incidentally found in the autopsy and did not give symptoms until death, not diagnosed despite the presence of chronic diseases is presented as a rare case which was not published in the literature before.

\section{REFERENCES}

[1] Turgutalp H., Intracranial cysts, Ankara Patology Bultellin. 15(2) Pp62-66 (1998)

[2] Ferreira T., Khurana D., Mohindra S., Gupta K.,Hemiatrophy as a presentation of a glioependymal cyst, ClinNeurolNeurosurg. 124 Pp6-7(2014)

[3] Osborn A. G., Preece M.T., İntracranial cysts: radiologic-pathologic correlation and imaging approach,Radiology.239(3) Pp650-664(2006)

[4] Boockvar J. A., Shafa R., Forman M. S., O'Rourke D. M., Symptomatic lateral ventricular ependymal cysts: criteria for distinguishing these rare cysts from other symptomatic cysts of the ventricles: case report,Neurosurgery.46(5)Pp1229-1233(2000)

[5] Zheng S. P., Ju Y., You C., Glioependymal cyst in children: a case report, ClinNeurol Neurosurg. 115 Pp2288-2290(2013)

[6] Friede R. L., Yasargil M. G., Supratentorial intracerebral epithelial (ependymal) cysts: review, case reports, and fine structure, $\mathrm{J}$ NeurolNeurosurg Psychiatry. 40 Pp127-137 (1977)

[7] Bouch D. C., Mitchell I., MaloneyA. F. J., Ependymal lined paraventricular cerebral cysts; a report of three cases, J NeurolNeurosurg Psychiatry. 36 Pp611-617(1973)

[8] Jakubiak P., Dunsmore R. H., Beckett R. H., Supratentorial brain cysts, J Neurosurg. 28(2) Pp129-136 (1968) 
[9] Niwa J., Okuyama T., Shimizu K., Hirai H., Glio-ependymal cyst in the lateral ventricle complicated by agenesis of the corpus callosum, No ShinkeiGeka. 19(4) Pp359-363 (1991)

[10] Obaldo R. E., Shao L., Lowe L. H., Congenital glioependymal cyst presenting with severe proptosis, Am J Neuroradiol. 28 Pp999-1000 (2007)

[11] Shukla-Davea A., GuptaaR. K., RoydR., HusaineN., PaulbL., VenkateshaS. K., RashidfM. R., ChhabracD. K., HusainM., Prospective evaluation of in vivo proton MR spectroscopy in differentiation of similar appearing intracranial cystic lesions, Magnetic Resonance Imaging. 19Pp103-110 (2001)
[12] Mühler M. R., Hartmann C., Werner W., Meyer O., Bollmann R., Klingebiel R., Fetal MRI demonstrates glioependymal cyst in a case of sonographic unilateral ventriculomegaly, Pediatric Radiology 37 Pp391-395 (2007)

[13] E. Pan, M. D. Prados, Holland-Frei Cancer Medicine. 6th ed. Hamilton (ON): BC Decker, 2003. ch. 83. Available from: https://www.ncbi. nlm.nih.gov/books/NBK13708/

[14] Chandana S. R., Movva S., Arora M., Singh T., Primary brain tumors in adults, American Family Physician. 77(10) Pp1423-1430 (2008)

Citation: Gizem Muratoglu, Ibrahim Ortanca, Muhammed Mustafa Arslan. Case Report: Postmortem Incidental Ependymal Cyst Opened to the Extracranial. ARC Journal of Forensic Science. 2017 2(1): 17-20. http://dx.doi.org/10.20431/2456-0049.0201005

Copyright: (C) 2017 Authors. This is an open-access article distributed under the terms of the Creative Commons Attribution License, which permits unrestricted use, distribution, and reproduction in any medium, provided the original author and source are credited. 